\title{
On the monotonicity of generalized barycentric coordinates on convex polygons
}

\author{
Michael S. Floater*
}

January 28, 2016

\begin{abstract}
We show that four well-known kinds of generalized barycentric coordinates in convex polygons share a simple monotonicity property: the coordinate function associated with a vertex is increasing along any line from the polygon boundary to that vertex. This shows that the coordinate functions have no local extrema and that their contours are single curves connecting pairs of points on the two edges adjacent to the vertex.

Keywords: monotonicity, Wachspress coordinates, harmonic coordinates, GordonWixom coordinates, mean value coordinates, GBCs.
\end{abstract}

\section{Introduction}

Let $P \subset \mathbb{R}^{2}$ be a convex polygon, with vertices $\mathbf{v}_{1}, \mathbf{v}_{2}, \ldots, \mathbf{v}_{n}, n \geq 3$, in some anticlockwise ordering. Figure 1 shows an example with $n=5$. Any functions

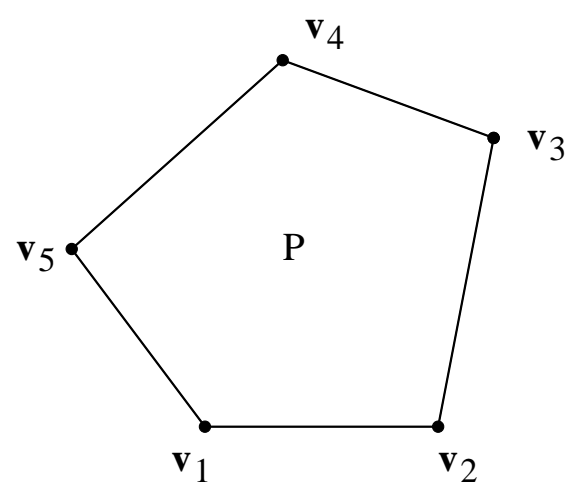

Figure 1: Convex polygon

*Department of Mathematics, University of Oslo, Moltke Moes vei 35, 0851 Oslo, Norway, email: michaelf@math.uio.no 
$\phi_{i}: P \rightarrow \mathbb{R}, i=1, \ldots, n$, are generalized barycentric coordinates (GBCs) if, for all $\mathbf{x} \in P, \phi_{i}(\mathbf{x}) \geq 0, i=1, \ldots, n$, and

$$
\sum_{i=1}^{n} \phi_{i}(\mathbf{x})=1, \quad \sum_{i=1}^{n} \phi_{i}(\mathbf{x}) \mathbf{v}_{i}=\mathbf{x} .
$$

From this definition one can show that all GBCs have the same values on the boundary of the polygon, $\partial P$. Specifically, $\left.\phi_{i}\right|_{\partial P}=f_{i}$, where the boundary function $f_{i}: \partial P \rightarrow \mathbb{R}$ has the values

$$
\begin{aligned}
\left.f_{i}\right|_{e_{j}} & =0, & & j \neq i-1,1, \\
f_{i}\left((1-\mu) \mathbf{v}_{i \pm 1}+\mu \mathbf{v}_{i}\right) & =\mu, & & \mu \in[0,1],
\end{aligned}
$$

and $e_{j}$ is the $j$-th edge, $e_{j}:=\left[\mathbf{v}_{j}, \mathbf{v}_{j+1}\right]$. Here and throughout, vertices, edges, and so on are indexed cyclically, i.e., $\mathbf{v}_{n+1}:=\mathbf{v}_{1}$ etc.

We can see that $\phi_{i}$ is increasing along the edges $e_{i-1}$ and $e_{i}$ in the direction towards $\mathbf{v}_{i}$. In this note we show that several well known GBCs share a more general monotonicity property. We will say that $\phi_{1}, \ldots, \phi_{n}$ are monotonic if, for all $i=$ $1, \ldots, n$, and for all $\mathbf{y} \in \partial P, \mathbf{y} \neq \mathbf{v}_{i}$, the coordinate $\phi_{i}$ is increasing along the line segment from $\mathbf{y}$ to $\mathbf{v}_{i}$. Figure 2 shows an example. Thus, by monotonic we mean

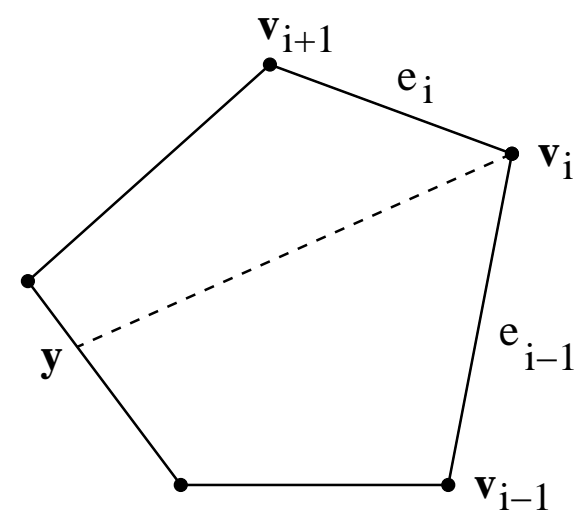

Figure 2: Line segment connecting a boundary point $\mathbf{y}$ to $\mathbf{v}_{i}$.

that if

$$
\mathbf{x}=(1-\lambda) \mathbf{y}+\lambda \mathbf{v}_{i} \quad \text { and } \quad \tilde{\mathbf{x}}=(1-\tilde{\lambda}) \mathbf{y}+\tilde{\lambda} \mathbf{v}_{i}
$$

with $0<\lambda<\tilde{\lambda}<1$, then $\phi_{i}(\mathbf{x})<\phi_{i}(\tilde{\mathbf{x}})$, and we need only check this property for boundary points $\mathbf{y} \notin e_{i-1}, e_{i}$. We will show that four distinct kinds of GBCs are monotonic: Wachspress coordinates, harmonic coordinates, Gordon-Wixom coordinates, and mean value coordinates.

Monotonicity does not follow from the definition (1) alone, since it is local to each point $\mathbf{x}$. We could construct an example of 'strange' GBCs that are neither continuous nor monotonic. If both coordinates of $\mathbf{x}$ are rational numbers, let $\phi_{1}(\mathbf{x}), \ldots, \phi_{n}(\mathbf{x})$ be the Wachspress coordinates of $\mathbf{x}$. Otherwise, if either coordinate is non-rational, let $\phi_{1}(\mathbf{x}), \ldots, \phi_{n}(\mathbf{x})$ be the mean value coordinates of $\mathbf{x}$. This defines a set of GBCs 
$\phi_{1}, \ldots, \phi_{n}$ for all $\mathbf{x}$ in $P$ that are clearly not continuous (except on the boundary of $P)$, and clearly not monotonic either.

The monotonicity property shows that each contour of $\phi_{i}$ is a single curve connecting a point on the edge $e_{i-1}$ to the corresponding point on the edge $e_{i}$. Despite the fact that numerous contour plots of GBCs have appeared in the literature over several years, and they tend to exhibit this behaviour, a mathematical proof seems to be missing. Another consequence of monotonicity is that $\phi_{i}$ cannot have any extrema in $P$.

It seems reasonable to use the simple term 'monotonic' because (a) this is the property that $\phi_{i}$ has along each line passing through $\mathbf{v}_{i}$ and (b) it seems unlikely that $\phi_{i}$ will be monotonic, in general, along any other line. Certainly, if we take any two points $\mathbf{y}_{1}$ and $\mathbf{y}_{2}$ on the boundary of $P$ that lie on distinct edges other than $e_{i}$ and $e_{i-1}$, then $\phi_{i}\left(\mathbf{y}_{1}\right)=\phi_{i}\left(\mathbf{y}_{2}\right)=0$ and $\phi_{i}$ is positive on the line between $\mathbf{y}_{1}$ and $\mathbf{y}_{2}$, from which we conclude that $\phi_{i}$ is not monotonic on that line.

\section{Wachspress' rational coordinates}

To define Wachspress' coordinates (Wachspress 1975), (Warren 1996), (Meyer, Barr, Lee \& Desbrun 2002), (Warren, Schaefer, Hirani \& Desbrun 2007), let $\mathbf{n}_{j}$ denote the outward unit normal to the edge $e_{j}$, and for $\mathbf{x} \in P$, let $h_{j}(\mathbf{x})$ be the perpendicular distance of $\mathbf{x}$ to $e_{j}$, i.e., by the scalar product,

$$
h_{j}(\mathbf{x})=\left(\mathbf{v}_{j}-\mathbf{x}\right) \cdot \mathbf{n}_{j}=\left(\mathbf{v}_{j+1}-\mathbf{x}\right) \cdot \mathbf{n}_{j} .
$$

Then the $i$-th Wachspress coordinate is $\phi_{i}=w_{i} / W$, where

$$
w_{i}(\mathbf{x})=\frac{c_{i}}{h_{i-1}(\mathbf{x}) h_{i}(\mathbf{x})}, \quad c_{i}=\mathbf{n}_{i-1} \times \mathbf{n}_{i}, \quad W=\sum_{j=1}^{n} w_{j},
$$

and $\times$ is the scalar-valued cross product, so $\mathbf{n}_{i-1} \times \mathbf{n}_{i}=\operatorname{det}\left[\mathbf{n}_{i-1}, \mathbf{n}_{i}\right]$.

To derive the monotonicity property consider the gradient $\nabla \phi_{i}$ of $\phi_{i}$ for some fixed $i$. By the quotient rule,

$$
\nabla \phi_{i}=\frac{1}{W^{2}}\left(W \nabla w_{i}-w_{i} \nabla W\right)=\frac{w_{i}}{W^{2}} \sum_{j=1}^{n} w_{j}\left(\mathbf{R}_{i}-\mathbf{R}_{j}\right),
$$

where $\mathbf{R}_{j}:=\nabla w_{j} / w_{j}$. Therefore, the directional derivative of $\phi_{i}$ in the (not necessarily unit) direction $\mathbf{v}_{i}-\mathbf{x}$ is

$$
D_{\mathbf{v}_{i}-\mathbf{x}} \phi_{i}(\mathbf{x})=\left(\mathbf{v}_{i}-\mathbf{x}\right) \cdot \nabla \phi_{i}(\mathbf{x})=\frac{w_{i}(\mathbf{x})}{W^{2}(\mathbf{x})} \sum_{j \neq i} w_{j}(\mathbf{x})\left(a_{i i}(\mathbf{x})-a_{i j}(\mathbf{x})\right),
$$

where

$$
a_{i j}(\mathbf{x}):=\left(\mathbf{v}_{i}-\mathbf{x}\right) \cdot \mathbf{R}_{j}(\mathbf{x}) .
$$

Thus to show that $D_{\mathbf{v}_{i}-\mathbf{x}} \phi_{i}(\mathbf{x})>0$, and since $w_{k}(\mathbf{x})>0$ for all $k$, it is sufficient to show that

$$
a_{i i}(\mathbf{x})>a_{i j}(\mathbf{x}), \quad j \neq i .
$$


Since $\nabla h_{j}=-\mathbf{n}_{j}$, we find

$$
\nabla w_{j}(\mathbf{x})=\left(\frac{\mathbf{n}_{j-1}}{h_{j-1}(\mathbf{x})}+\frac{\mathbf{n}_{j}}{h_{j}(\mathbf{x})}\right) w_{j}(\mathbf{x})
$$

and so

$$
\mathbf{R}_{j}(\mathbf{x})=\frac{\mathbf{n}_{j-1}}{h_{j-1}(\mathbf{x})}+\frac{\mathbf{n}_{j}}{h_{j}(\mathbf{x})}
$$

as shown in (Floater, Gillette \& Sukumar 2014). Now observe that for any $k=$ $1, \ldots, n$,

$$
\left(\mathbf{v}_{i}-\mathbf{x}\right) \cdot \mathbf{n}_{k}=\left(\mathbf{v}_{k}-\mathbf{x}\right) \cdot \mathbf{n}_{k}-\left(\mathbf{v}_{k}-\mathbf{v}_{i}\right) \cdot \mathbf{n}_{k}=h_{k}(\mathbf{x})-h_{k}\left(\mathbf{v}_{i}\right),
$$

and therefore, for any $j=1, \ldots, n$,

$$
a_{i j}(\mathbf{x})=2-\frac{h_{j-1}\left(\mathbf{v}_{i}\right)}{h_{j-1}(\mathbf{x})}-\frac{h_{j}\left(\mathbf{v}_{i}\right)}{h_{j}(\mathbf{x})} .
$$

It follows that $a_{i i}(\mathbf{x})=2$ and $a_{i j}(\mathbf{x})<2, j \neq i$, which implies (5).

\section{Harmonic coordinates}

The harmonic coordinates (Floater, Hormann \& Kós 2006), (Joshi, Meyer, DeRose, Green \& Sanocki 2007) are defined by the Laplace equation with Dirichlet boundary condition:

$$
\begin{aligned}
\Delta \phi_{i} & =0, \quad \text { in } P, \\
\phi_{i} & =f_{i}, \quad \text { on } \partial P .
\end{aligned}
$$

Here, $\Delta$ is the Laplace operator, $\partial^{2} / \partial x^{2}+\partial^{2} / \partial y^{2}$, where $\mathbf{x}=(x, y)$.

To show monotonicity, it is sufficient to show that $\psi_{i}>0$ in the interior of $P$, where

$$
\psi_{i}(\mathbf{x}):=D_{\mathbf{v}_{i}-\mathbf{x}} \phi_{i}(\mathbf{x})=\left(\mathbf{v}_{i}-\mathbf{x}\right) \cdot \nabla \phi_{i}(\mathbf{x}), \quad \mathbf{x} \in P .
$$

To show this we can use the maximum principle (Protter \& Weinberger 1967) for the Laplace operator. By differentiating $\psi_{i}$ we find

$$
\Delta \psi_{i}=-2 \Delta \phi_{i}+\left(\mathbf{v}_{i}-\mathbf{x}\right) \cdot \nabla\left(\Delta \phi_{i}\right),
$$

and therefore, since $\phi_{i}$ is harmonic, so is $\psi_{i}$. Considering the boundary values of $\psi_{i}$, if $\mathbf{x}$ belongs to $e_{i \pm 1}$ then

$$
\psi_{i}(\mathbf{x})=\frac{\left|\mathbf{v}_{i}-\mathbf{x}\right|}{\left|\mathbf{v}_{i \pm 1}-\mathbf{v}_{i}\right|} \geq 0 .
$$

If, on the other hand, $\mathbf{x}$ belongs to an edge $e_{j}, j \neq i-1, i$, then, since $\phi_{i}(\mathbf{x})=0$ and $\phi_{i} \geq 0$ in $P$, we see that, by the convexity of $P, \psi_{i}(\mathbf{x}) \geq 0$. Thus $\psi_{i} \geq 0$ on $\partial P$. Since $\Delta \psi_{i}=0$ in $P$, and $\psi_{i}$ cannot be constant, it cannot attain its minimum in the interior of $P$, and so $\psi_{i}>0$ in $P$. 


\section{Gordon-Wixom coordinates}

To define Gordon-Wixom coordinates (Gordon \& Wixom 1974), (Belyaev 2006), (Manson, Li \& Schaefer 2011) let $\mathbf{x} \in P$ and for any angle $\theta \in \mathbb{R}$, let $\mathbf{y}_{\theta}$ be the unique point of intersection between the ray

$$
L_{\theta}:=\{\mathbf{x}+s(\cos \theta, \sin \theta): s \geq 0\}
$$

and the polygon boundary $\partial P$, and let $s_{\theta}=\left|\mathbf{y}_{\theta}-\mathbf{x}\right|$, the Euclidean distance from $\mathbf{x}$ to $\mathbf{y}_{\theta}$. The ray $L_{\theta+\pi}$, in the direction opposite to $L_{\theta}$, also meets $\partial P$ uniquely, at the point $\mathbf{z}_{\theta}:=\mathbf{y}_{\theta+\pi}$, whose distance from $\mathbf{x}$ is $t_{\theta}:=s_{\theta+\pi}$. Then the $i$-th coordinate at $\mathbf{x}$ is defined as the integral mean of linear interpolants,

$$
\phi_{i}(\mathbf{x})=\frac{1}{\pi} \int_{0}^{\pi}\left(\frac{s_{\theta}}{t_{\theta}+s_{\theta}} f_{i}\left(\mathbf{z}_{\theta}\right)+\frac{t_{\theta}}{t_{\theta}+s_{\theta}} f_{i}\left(\mathbf{y}_{\theta}\right)\right) d \theta,
$$

or equivalently,

$$
\phi_{i}(\mathbf{x})=\frac{1}{\pi} \int_{0}^{2 \pi} \frac{t_{\theta}}{t_{\theta}+s_{\theta}} f_{i}\left(\mathbf{y}_{\theta}\right) d \theta .
$$

To show monotonicity, suppose that $\mathbf{y}, \mathbf{x}, \tilde{\mathbf{x}}, \lambda$, and $\tilde{\lambda}$ are as in (3), and define $\tilde{\mathbf{y}}_{\theta}$, $\tilde{s}_{\theta}$, and $\tilde{t}_{\theta}$ with respect to the point $\tilde{\mathbf{x}}$, in the same way as $\mathbf{y}_{\theta}, s_{\theta}$, and $t_{\theta}$ are defined with respect to $\mathbf{x}$. Then, from (7), it is sufficient to show that

$$
\frac{t_{\theta}}{t_{\theta}+s_{\theta}} f_{i}\left(\mathbf{y}_{\theta}\right) \leq \frac{\tilde{t}_{\theta}}{\tilde{t}_{\theta}+\tilde{s}_{\theta}} f_{i}\left(\tilde{\mathbf{y}}_{\theta}\right)
$$

for all $\theta$, with strict inequality for at least one $\theta$. Suppose first that $\mathbf{y}_{\theta} \notin e_{i-1}, e_{i}$. Then $f_{i}\left(\mathbf{y}_{\theta}\right)=0$ and (8) trivially holds. Suppose otherwise that $\mathbf{y}_{\theta} \in e_{j}$ for some $j \in\{i-1, i\}$. Referring to Figure 3 , since $\tilde{\lambda} \in[\lambda, 1]$, we have $\tilde{\mathbf{x}} \in\left[\mathbf{x}, \mathbf{v}_{i}\right]$ and $\tilde{\mathbf{y}}_{\theta} \in\left[\mathbf{y}_{\theta}, \mathbf{v}_{i}\right]$ and $f_{i}\left(\mathbf{y}_{\theta}\right) \leq f_{i}\left(\tilde{\mathbf{y}}_{\theta}\right) \leq 1$.

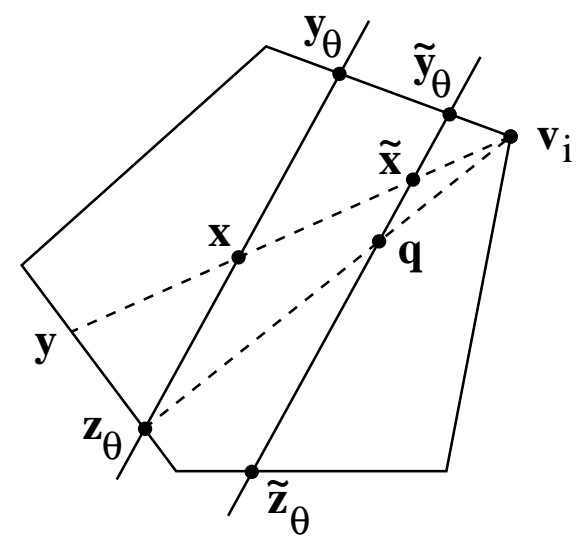

Figure 3: Points involved in Gordon-Wixom coordinates.

So $f_{i}\left(\mathbf{y}_{\theta}\right) \leq f_{i}\left(\tilde{\mathbf{y}}_{\theta}\right)$ and (8) holds if

$$
\frac{t_{\theta}}{t_{\theta}+s_{\theta}} \leq \frac{\tilde{t}_{\theta}}{\tilde{t}_{\theta}+\tilde{s}_{\theta}}
$$


or equivalently (by considering the reciprocal),

$$
\frac{t_{\theta}}{s_{\theta}} \leq \frac{\tilde{t}_{\theta}}{\tilde{s}_{\theta}}
$$

The distance $\tilde{s}_{\theta}$ decreases linearly with $\tilde{\lambda}$ for $\tilde{\lambda} \in[\lambda, 1]$, from $s_{\theta}$ to 0 , and so

$$
\tilde{s}_{\theta}=\frac{1-\tilde{\lambda}}{1-\lambda} s_{\theta}
$$

On the other hand, by the convexity of $P$, the line segment $\left[\mathbf{z}_{\theta}, \mathbf{v}_{i}\right]$ intersects the line segment $\left[\tilde{\mathbf{x}}, \tilde{\mathbf{z}}_{\theta}\right]$. Let $\mathbf{q}$ be the point of intersection, shown in Figure 3 . Then

$$
\tilde{t}_{\theta}=\left|\tilde{\mathbf{z}}_{\theta}-\tilde{\mathbf{x}}\right| \geq|\mathbf{q}-\tilde{\mathbf{x}}|=\frac{1-\tilde{\lambda}}{1-\lambda} t_{\theta},
$$

which, combined with (10), implies (9). This proves (8). We can also see that if $\mathbf{y}_{\theta} \in e_{j}$ for some $j \in\{i-1, i\}$ and $\mathbf{y}_{\theta} \neq \mathbf{v}_{i}$ then $f_{i}\left(\mathbf{y}_{\theta}\right)<f_{i}\left(\tilde{\mathbf{y}}_{\theta}\right)$ and the inequality in (8) is strict.

\section{Mean value coordinates}

The $i$-th mean value coordinate at $\mathrm{x} \in P$ (Floater 2003) can be defined as the ratio of integrals

$$
\phi_{i}(\mathbf{x})=\int_{0}^{2 \pi} \frac{f_{i}\left(\mathbf{y}_{\theta}\right)}{s_{\theta}} d \theta / \int_{0}^{2 \pi} \frac{1}{s_{\alpha}} d \alpha,
$$

with $\mathbf{y}_{\theta}$ and $s_{\theta}$ as defined in Section 4.

Regarding monotonicity, let $\mathbf{y}, \mathbf{x}$, and $\lambda$ be as in (3). To show that $\phi_{i}(\mathbf{x})$ is increasing in $\lambda$ at $\mathbf{x}$ it is sufficient to show that

$$
\frac{f_{i}\left(\mathbf{y}_{\theta}\right)}{s_{\theta}} / \int_{0}^{2 \pi} \frac{1}{s_{\alpha}} d \alpha
$$

is non-decreasing in $\lambda$ for all $\theta$, and increasing for some $\theta$. Then, since the reciprocal of an increasing function is decreasing, and vice versa, a similar consideration of the angle $\alpha$ shows that it is sufficient that

$$
\frac{s_{\alpha}}{s_{\theta}} f_{i}\left(\mathbf{y}_{\theta}\right)
$$

is non-decreasing for all $\theta$ and $\alpha$, and increasing for at least one choice of $\theta$. Thus it is sufficient to show that with $\tilde{\mathbf{x}}$ also as in (3),

$$
\frac{s_{\alpha}}{s_{\theta}} f_{i}\left(\mathbf{y}_{\theta}\right) \leq \frac{\tilde{s}_{\alpha}}{\tilde{s}_{\theta}} f_{i}\left(\tilde{\mathbf{y}}_{\theta}\right)
$$

for all $\theta$ and $\alpha$, with strict inequality for some choice of $\theta$. 
The proof of (13) is similar to that of (8). If $\mathbf{y}_{\theta} \notin e_{i-1}, e_{i}$ then (13) trivially holds because $f_{i}\left(\mathbf{y}_{\theta}\right)=0$. Otherwise, with $\mathbf{y}_{\theta} \in e_{j}$ for some $j \in\{i-1, i\}$, it is sufficient to show that

$$
\frac{s_{\alpha}}{s_{\theta}} \leq \frac{\tilde{s}_{\alpha}}{\tilde{s}_{\theta}} .
$$

This follows from equation (10) and the fact that, in analogy to (11),

$$
\tilde{s}_{\alpha} \geq \frac{1-\tilde{\lambda}}{1-\lambda} s_{\alpha},
$$

with $\mathbf{y}_{\alpha}$ and $\tilde{\mathbf{y}}_{\alpha}$ playing the role of $\mathbf{z}_{\theta}$ and $\tilde{\mathbf{z}}_{\theta}$, and this establishes (13). Similar to Section 4 , if $\mathbf{y}_{\theta} \in e_{j}$ for some $j \in\{i-1, i\}$ but with $\mathbf{y}_{\theta} \neq \mathbf{v}_{i}$ then $f_{i}\left(\mathbf{y}_{\theta}\right)<f_{i}\left(\tilde{\mathbf{y}}_{\theta}\right)$ and the inequality in (13) is strict.

\section{$6 \quad$ Higher dimensions}

As one might expect, the montonicity property studied here extends in the obvious way to convex polyhedra and, more generally, convex polytopes in higher dimensions for the four coordinates considered here, if we make the right definitions.

Considering first Wachspress coordinates, the proof of monotonicity of Section 2 generalizes at least to simple convex polytopes in $\mathbb{R}^{d}$, i.e., convex polytopes in which every vertex has $d$ adjacent $(d-1)$-dimensional faces. Using the definitions derived in (Warren et al. 2007), and applying the notation and gradient formulas of Sec. 2 of (Floater et al. 2014), the argument is similar, and inequality (5) now follows from the fact that $a_{i i}(\mathbf{x})=d$ and $a_{i j}(\mathbf{x})<d, j \neq i$.

For the remaining three kinds of GBCs: harmonic, GW, and MV, we do not need to restrict to a simple polytope, and, moreover, we have a choice of how to generalize the boundary functions $f_{i}$ of $(2)$. For each $(d-1)$-dimensional face of the convex polytope we could choose any set of GBCs for that face that are themselves monotonic. These face GBCs then define the boundary functions $f_{i}$ for the vertices $\mathbf{v}_{i}$ of the polytope. The proof of monotonicity of the harmonic coordinates is now as before, with $\psi_{i}$ harmonic in $P$ and $\psi_{i} \geq 0$ on $\partial P$ due to $f_{i}$ being monotonic on the faces adjacent to $\mathbf{v}_{i}$. The proof of monotonicity for GW and MV coordinates is also similar to the $2-\mathrm{D}$ case, with integration carried out over unit vectors $\boldsymbol{\mu}$ on the unit sphere in $\mathbb{R}^{d}$ instead of angles around the unit circle. We would replace the ray $L_{\theta}$ of (6) by

$$
L_{\boldsymbol{\mu}}:=\{\mathbf{x}+s \boldsymbol{\mu}: s \geq 0\},
$$

and could denote by $\mathbf{y}_{\boldsymbol{\mu}}$ its point of intersection with $\partial P$. Then for any fixed $\boldsymbol{\mu}$, the five points $\mathbf{v}_{i}, \mathbf{x}$, and $\tilde{\mathbf{x}}$ of (3) and $\mathbf{y}_{\boldsymbol{\mu}}$ and $\tilde{\mathbf{y}}_{\boldsymbol{\mu}}$ would all lie in the same plane, and the steps used to prove (8) and (13) are the same.

\section{Future work}

Monotonicity could also be investigated for other GBCs, such as maximum entropy coordinates (Sukumar 2004), the families of coordinates of (Floater et al. 2006), and the inverse rational bilinear coordinates for quadrilaterals of (Floater 2015). 
A shape-property related to monotonicity is convexity. Are there GBCs which are convex as well as monotonic along the lines considered here?

Acknowledgement. I wish to thank the three referees and the editor for their careful reading of the manuscript and their valuable comments which have helped to improve the paper.

\section{References}

Belyaev, A. (2006), On transfinite barycentric coordinates, in 'Symposium on Geometry Processing 2006', Eurographics Association, pp. 89-99.

Floater, M. S. (2003), 'Mean value coordinates', Comput. Aided Geom. Design 20, 19-27.

Floater, M. S. (2015), 'The inverse of a rational bilinear mapping', Comput. Aided Geom. Design 33, 46-50.

Floater, M. S., Gillette, A. \& Sukumar, N. (2014), 'Gradient bounds for Wachspress coordinates on polytopes', SIAM J. Numer. Anal. 52, 515-532.

Floater, M. S., Hormann, K. \& Kós, G. (2006), 'A general construction of barycentric coordinates over convex polygons', Adv. Comput. Math. 24, 311-331.

Gordon, W. J. \& Wixom, J. A. (1974), 'Pseudo-harmonic interpolation on convex domains', SIAM J. Numer. Anal. 11, 909-933.

Joshi, P., Meyer, M., DeRose, T., Green, B. \& Sanocki, T. (2007), 'Harmonic coordinates for character articulation', ACM Trans. on Graph. 26.

Manson, J., Li, K. \& Schaefer, S. (2011), 'Positive Gordon-Wixom coordinates', Computer Aided Design 43, 1422-1426.

Meyer, M., Barr, A., Lee, H. \& Desbrun, M. (2002), 'Generalized barycentric coordinates for irregular polygons', J. Graph. Tools 7, 13-22.

Protter, M. H. \& Weinberger, H. F. (1967), Maximum principles in differential equations, Prentice-Hall, N.J.

Sukumar, N. (2004), 'Construction of polygonal interpolants: a maximum entropy approach', Int. J. Numer. Meth. Engng 61, 2159-2181.

Wachspress, E. L. (1975), A Rational Finite Element Basis, Academic Press, New York.

Warren, J. (1996), 'Barycentric coordinates for convex polytopes', Adv. Comput. Math. 6, 97-108.

Warren, J., Schaefer, S., Hirani, A. N. \& Desbrun, M. (2007), 'Barycentric coordinates for convex sets', Adv. Comput. Math. 27, 319-338. 